\title{
Quantifying Missingness in Wearable Heart Rate Recordings
}

\author{
Tim COLLINS ${ }^{\mathrm{a}, 1}$, Sandra I. WOOLLEY ${ }^{\mathrm{b}}$, Salome ONIANI $^{\mathrm{c}}$ and Anand PANDYAN ${ }^{\mathrm{b}}$ \\ ${ }^{a}$ Manchester Metropolitan University, UK \\ ${ }^{\mathrm{b}}$ Keele University, UK \\ ' Ilia State University, Georgia
}

\begin{abstract}
Wrist-worn photoplethysmography (PPG) heart rate monitoring devices are increasingly used in clinical applications despite the potential for data missingness and inaccuracy. This paper provides an analysis of the intermittency of experimental wearable data recordings. Devices recorded heart rate with gaps of 5 or more minutes $41.6 \%$ of the time and 15 or more minutes $3.8 \%$ of the time.
\end{abstract}

Keywords. wearable, heart rate, photoplethysmography, missingness

\section{Introduction}

The clinical application of wearable consumer-grade PPG heart rate monitors is evidenced by 601 'fitbit' search results from the ClinicalTrials.gov database [1]. However, reliable heart rate estimation from on-wrist PPG sensors is challenging [2,3], particularly during periods of activity [4]. Embedded device firmware can attempt to identify and reject less reliable readings, but this can introduce gaps in recorded data.

\section{Methods}

We extracted 1280 minutes of recorded data from Garmin Vivosmart 3 (v4.10).FIT files. These were recorded from four participants (P01-4), wearing four devices (D01-4), two per wrist, during 80-minute treadmill walking activities comprising 20 minutes at each of $2.4,3.2,4.8$ and $6.4 \mathrm{~km} / \mathrm{h}$. We compared recordings and timestamps made by Vivosmart 3 devices and a Polar H10 chest strap ECG monitor (1W v2.1.9).

\section{Results}

The Vivosmart 3 results are summarized in figure $1.37 .9 \%$ of the 1280 minutes of heart rate recordings were made at 1 -minute intervals. $41.6 \%$ were made at intervals of 5 or more minutes. $12.3 \%$ were made at 10 or more minute intervals and $3.8 \%$ were made at 15 or more minute intervals. Mean Absolute Percentage Errors (MAPEs) between 4.34\%

${ }^{1}$ Corresponding Author, Tim Collins, Department of Engineering, Manchester Metropolitan University, Manchester, M1 5GD; E-mail: T.Collins@mmu.ac.uk 
and $16.00 \%$ were previously reported for this data [4,5]; in analyzing the intervals between these recordings we found no significant positive or negative correlations between heart rate estimation errors and the time between recordings.
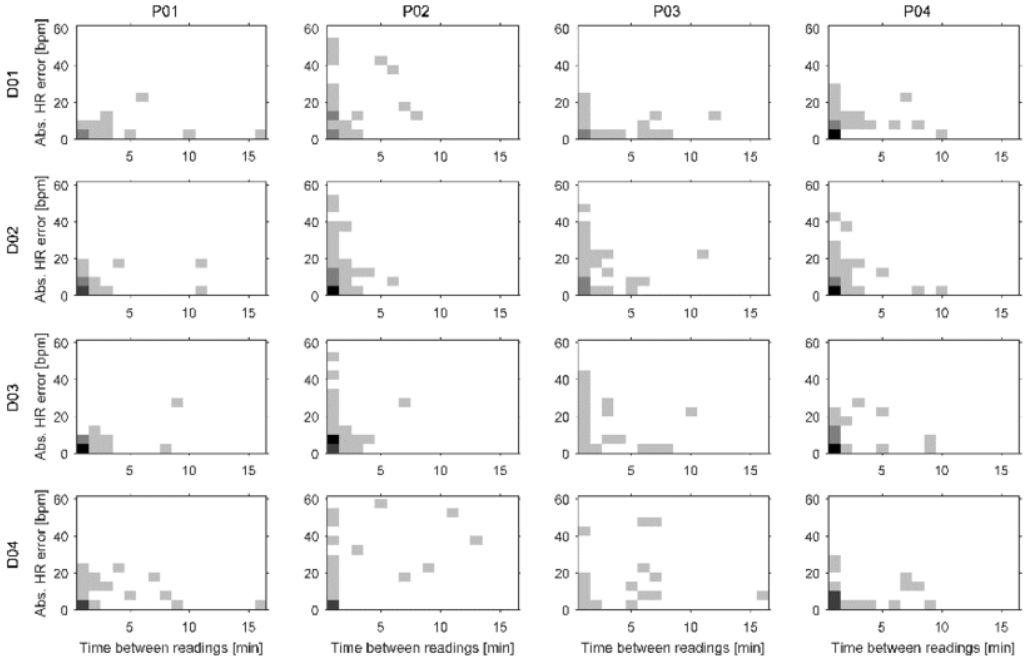

No. of occurrences:

$\square 6-10$

Figure 1. Heat maps showing the frequency of absolute heart rate errors and times between recordings

\section{Discussion}

Data missingness is not widely reported in the literature but it is important if real-time physiological data recording is significant to clinical application or patient monitoring.

\section{Conclusions}

We have quantified data missingness in wearable device heart rate estimates and demonstrated the intermittent nature of device recordings and the long delays that can occur between logged heart rate estimates.

\section{References}

[1] U.S. National Library of Medicine, "Clinicaltrials.gov," 2020, Available at https://www.clinicaltrials.gov/, 1Accessed Dec 28, 2020.

[2] Collins T, Pires I, Oniani S, Woolley S. How reliable is your wearable heart rate monitor? The Conversation, Health+Medicine, June 2018.

[3] Bent B, Goldstein BA, Kibbe WA, Dunn JP. Investigating sources of inaccuracy in wearable optical heart rate sensors. npj Digital Medicine; 2020 Feb:3(1).

[4] Collins T, Woolley SI, Oniani S, Pires IM, Garcia NM, Ledger SJ, Pandyan A. Version Reporting and Assessment Approaches for New and Updated Activity and Heart Rate Monitors. Sensors (Basel). 2019 Apr 10;19(7):1705.

[5] Oniani S, Woolley SI, Pires IM, Garcia NM, Collins T, Ledger S, Pandyan A. Reliability assessment of new and updated consumer-grade activity and heart rate monitors. in SensorDevices. 2018. 